\title{
Syncope as a health risk for soldiers - influence of medical history and clinical findings on the sensitivity of head-up tilt table testing
}

Hans-Joachim Gilfrich', Lena Marie Heidelmann², Franziska Grube², Hagen Frickmann ${ }^{3,4^{*}+}$ and Sven Andreas Jungblut ${ }^{1+}$

\begin{abstract}
Background: Syncope is a relevant health problem in military environments. Reliable diagnosis is challenging. Tilt table testing is an important tool for syncope diagnosis. The aim of this study was to determine whether signs such as prodromal symptoms, co-morbidity, frequency of syncopal events, body length, body mass index, and electrocardiography abnormalities can be used to predict the success of tilt table testing at diagnosing syncope.

Methods: Data from 100 patients with histories of syncope or pre-syncope, who were diagnosed using head-up tilt table testing, were retrospectively analyzed in a cross-sectional analysis. The diagnostic procedure was based upon a modified version of the Westminster protocol without any pharmacological provocation.

Results: Patients showing pathological reaction patterns during tilt table testing suffered from prodromal symptoms, such as dizziness and sweating, significantly more often. The patients reported more injuries resulting from syncopal events and more previous syncopal events, and the prevalence of co-morbidity was greater among patients presenting negative findings during tilt testing. An asthenic-leptosomal physique was not confirmed as a risk factor for syncopal events as is the case for idiopathic arterial hypotension. However, patients with pathological reaction patterns during tilt table testing were significantly taller. This finding was detected for both females and males. No significant predictors were found in the electrocardiogram (ECG) patterns of patients showing syncope during tilt table testing.

Conclusions: Frequency of prior syncope and prodromal symptoms, and increased body length with an otherwise good state of health influence the predictive value of tilt table testing for syncope diagnosis. In particular, if these factors are present, tilt table testing should be considered part of the diagnostic algorithm for soldiers with recurrent syncope.
\end{abstract}

Keywords: Syncope, Head-up tilt testing, Predictor, Soldiers, Assessment, Hypotension

\section{Background}

Syncope has been defined as a transient, self-limited loss of consciousness based on a transient global cerebral hypoperfusion that usually leads to falling with rapid onset and spontaneous subsequent complete and prompt recovery [1]. As with civilians, military service members

\footnotetext{
* Correspondence: frickmann@bni-hamburg.de

${ }^{\dagger}$ Equal contributors

${ }^{3}$ The Department of Tropical Medicine at the Bernhard Nocht Institute,

German Armed Forces Hospital of Hamburg, Hamburg, Germany

${ }^{4}$ The Department of Microbiology, Virology and Hygiene, University Medicine Rostock, Rostock, Germany

Full list of author information is available at the end of the article
}

can be affected. Syncope commonly affects personnel that stand in ranks during military ceremonies, leading to disruption of the ceremonies and embarrassment for the individuals involved [2]. The identification of a possible sudden incapacitation during military flight is essential to maintain high aviation safety. Sudden incapacitation can be associated with syncope [3]. Medical assessment, including an evaluation of incapacitation risk, is mandatory for flight personnel who wish to obtain the medical certificate that is required for maintaining pilot licenses in European aviation [3, 4].

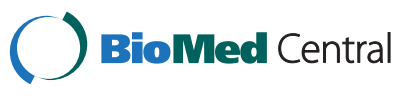

(c) 2015 Gilfrich et al. Open Access This article is distributed under the terms of the Creative Commons Attribution 4.0 International License (http://creativecommons.org/licenses/by/4.0/), which permits unrestricted use, distribution, and reproduction in any medium, provided you give appropriate credit to the original author(s) and the source, provide a link to the Creative Commons license, and indicate if changes were made. The Creative Commons Public Domain Dedication waiver (http://creativecommons.org/publicdomain/zero/1.0/) applies to the data made available in this article, unless otherwise stated. 
The calculated incidence rate of syncope within the armed forces of the United States of America is 7.2 cases per 1,000 person years. Four percent of syncopal events are associated with physical injury. Frequency of syncope in association with vaccination ranges from 4.4 to 14.1 events per 100,000 immunization episodes [5].

Head-up tilt testing is the gold standard for the diagnosis of neurocardiogenic syncope (also called vasovagal syncope). The procedure allows five types of reaction patterns to be distinguished: neurocardiogenic syncope (including vaso-depressive, cardio-inhibitory and mixed subtypes), dysautonomic syncope, postural tachycardia syndrome (POTS), cerebral syncope, and psychogenic syncope [6].

However, head-up tilt testing remains a technically demanding and time-consuming diagnostic procedure despite its advantages [7, 8]. Thus, it is not routinely performed to diagnose syncopal events, although the pathophysiological background of approximately half of etiologically unclear syncopal events can be determined using head-up tilt testing [9]. Accordingly, the search for predictors of the efficiency of using this method for the differential diagnosis of syncope appears useful.

This retrospective assessment was performed to identify features in the clinical history or diagnostic findings that can help to identify patients with syncope who are likely to obtain diagnostic benefit from head-up tilt testing. Parameters including prodromal symptoms, accompanying diseases, frequency of syncope, height, body mass index, and electrocardiogram(ECG)-findings were analyzed to assess their prognostic value for the onset of syncopal events during head-up tilt testing.

Although some parameters (e.g., height) are known factors for tolerance time to tilt, literature results are partly inconsistent, as detailed in the discussion section. This proof-of-principle analysis was designed to observe whether such factors are reproducible and to strengthen described associations based on additional data. In this way, the aim of this study was to identify major and minor criteria that might suggest diagnostic benefit from head-up tilt testing that might be worth assessing in sufficiently powered future studies.

\section{Methods}

\section{Patients}

Data obtained from 100 consecutive patients (45 male, 55 female) were anonymously and retrospectively assessed in this cross-sectional assessment. The mean age of the population $( \pm$ SD) was 53 ( \pm 19 years), and the median age was 56 . The diagnostic setting was previously described in detail [10] and is summarized below (Table 1).

The youngest patient was 15 years, and the oldest was 88 years. All patients were referred to the hospital due to a history of suspected neurocardiogenic syncope.
Table 1 Patient clinical data

\begin{tabular}{ll}
\hline Item & Data \\
\hline Total number of patients & 100 \\
Male $[n(\%)]$ & 45 (45\%) \\
Female $[n(\%)]$ & $55(55 \%)$ \\
Average age (year) & 53 \\
Minimum/maximum age (year) & $15 / 88$ \\
Syncopal events & \\
Minimum & 0 \\
Maximum & 40 \\
Mean value & 4.43 \\
Standard deviation & 5.98 \\
Patients without medical history of syncope & 12 \\
Accompanying diseases & \\
Arterial hypertonus & 19 \\
Diabetes mellitus & 9 \\
Atherosclerosis of the carotic blood vessels and/or \\
central nervous system blood vessels \\
Coronary heart disease \\
$\begin{array}{l}\text { Disturbed heart rhythm } \\
\text { Diseases of the thyroid gland } \\
\text { Heart insufficiency New York Heart Association I-II }\end{array}$ \\
\hline
\end{tabular}

History of syncope or pre-syncope was the main criterion for inclusion. Exclusion criteria included the following illnesses: hypertrophic obstructive cardiomyopathy, higher-grade stenosis of the aorta, pulmonary hypertonus, bradycardia (sick sinus syndrome, second- or third-grade atrioventricular block), tachycardia (supraventricular tachycardia, ventricular tachycardia, Torsadede-pointes-tachycardia), haemodynamically relevant stenoses of the central blood vessels, heart insufficiency of the New York Heart Association stages III-IV, and neurological disorders that can cause disturbed orthostatic regulation (Shy-Drager's syndrome, Parkinson's disease, Encephalitis disseminata, Guillain-Barrés syndrome, and narcolepsy).

The frequency of syncopal events ranged between a minimum of zero and a maximum of 40 syncope events. The mean value was approximately $4.43 \pm 5.94$ (SD) events. Twelve patients were free of proven syncopal events. These patients showed pre-syncope or typical symptoms of disturbed orthostatic regulation.

The following accompanying diseases were present: 19 patients had arterial hypertonus, 9 patients had type II diabetes mellitus, 9 patients had atherosclerosis of the carotic blood vessels and/or blood vessels of the central nervous system, 8 patients had coronary heart disease, 7 patients had known disturbances of the heart rhythm, 6 patients had diseases of the thyroid gland and 4 patients had a heart insufficiency of a lesser grade than 
New York Heart Association III. Approximately 54 patients did not present any accompanying disease (Table 1).

All patients were asked about typical prodromal symptoms before the onset of syncopal events. In particular, the incidence of vertigo, nausea, black eyesight, sweating, headache or head pressure, tachycardia, bradycardia, and injuries was determined. The height and body mass index of all study participants were measured. An electrocardiogram with 12 channels was obtained using a MAC 1200 ST ECG device (GE Healthcare, Chalfont St Giles, UK), and a routine blood analysis was performed. Blood was drawn in the morning prior to head-up tilt table testing.

\section{Test conditions}

Using the head-up tilt device (which was electronically controlled via a hydraulic system), the intended tilt angle could be adjusted and returned to the starting position within 15-20 seconds. A remote control device allowed the intended tilt angle to be programmed directly. The tilt could be controlled using an external protractor that was attached to the table. The head-up tilt device was self-designed as described [10]. Electrocardiographic (ECG) analysis was continuously performed throughout the analysis. The ECG analysis was recorded using chart paper. Blood pressure was measured in parallel according to Riva-Rocci [11]. Blood pressure cuffs (Erkarapid classic Germany; Erka. Kallmeyer Medizintechnik GmbH \& Co. KG, Bad Tölz, Germany) and Littmann classic II S.E. stethoscopes (3 M Deutschland GmbH, Neuss, Germany) were used. The test was performed in a warm, quiet room under dimmed light to avoid unintended stimulation of the nervous system. The testing took place between 9 and 12 AM. The patients were asked to abstain from food and liquids for at least 6 hours prior to the test to avoid postprandial orthostatic hypotonia. Nicotine consumption was also prohibited for 6 hours prior to the testing. The administration of cardiovascular-acting drugs was ceased for at least 5 half lives of the respective drug before the testing. All patients were informed about the procedure; a standardized questionnaire and a detailed talk were presented to prepare for the testing.

The applied head-up tilt testing protocol was based upon a modified version [12] of the "Westminster protocol" [7], the first recommended protocol for medical head-up tilt testing. After fixing the patient at the table and applying electrocardiography electrodes and blood measurement equipment, the patients were allowed to rest for twenty minutes in a horizontal position. An electrocardiography sheet was printed at the end of the rest period. The patient was moved within 15 seconds during the following tilt period into a $70^{\circ}$ angle in relation to the initial position, with the head up. An additional electrocardiography sheet was printed one minute after the new position was reached. This test was ended after 45 minutes if no symptoms occurred. Finally, the patient was then returned to the horizontal position and allowed to rest for five minutes. A further electrocardiography sheet was printed at the end of the analysis. In cases of syncope or pre-syncope, the patients were returned immediately to the horizontal position. In such cases, electrocardiography sheets were printed continuously until the cardiovascular parameters returned to normal values.

Patients who suffered from neurocardiogenic or orthostatic syncope during the head-up tilt testing were compared with patients who remained asymptomatic during the procedure. Patients with postural tachycardia syndrome during head-up tilt testing were not included in either of the two groups to guarantee clear discrimination.

\section{Descriptive statistics}

All results were described using representative parameters, such as median, mean, and standard deviation. The quantitative results of the groups were compared using Student's $t$-test. The measured values were subjected to normality testing as a prerequisite for t-testing. Nonparametric (Mann-Whitney) testing was performed in case normality could not be confirmed. Values of $P<0.05$ were considered significant. For data obtained using contingency tables, Fischer's exact test with Yate's continuity correction was applied to calculate two-sided $P$ values. Values of $P<0.05$ were considered significant.

\section{Ethical considerations}

Ethical clearance was not required for this assessment in accordance with German law. Anonymous patient data were retrospectively assessed. No medical procedures were performed for study purposes. Accordingly, the project did not fulfill the criteria for description as a scientific project involving humans according to $\mathbb{\$} 9.2$ of the Law of the Association of Hamburg Physicians ("Hamburgisches Kammergesetz für Heilberufe"), and ethical counseling was not required according to $\mathbb{} 15.1$ of the Professional Guidelines of Hamburg Physicians ("Berufsordnung für Hamburger Ärzte und Ärztinnen"). Due to the anonymous nature of the assessment, German data protection law ("Bundesdatenschutzgesetz") was not applicable.

\section{Results}

\section{Results of head-up tilt testing}

Head-up tilt testing of 100 patients was assessed; of these, 56 patients showed a non-pathological result. Fourteen patients suffered from orthostatic dysautonomia with syncope during the test. Neurocardiogenic syncope was 


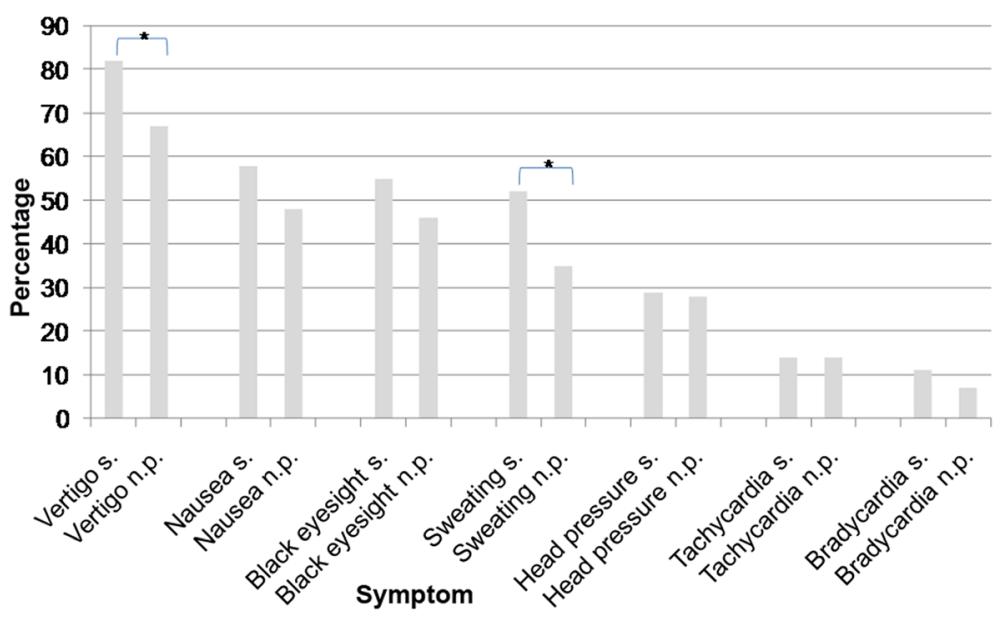

Fig. 1 Prodromal symptoms. s. Syncope during head-up tilt testing; n.p. No pathological findings during head-up tilt testing. ${ }^{*} P<0.05$

detected in 20 patients. An additional 10 patients presented postural tachycardia syndrome (POTS).

In summary, syncopal events were induced in $34 \%$ of the patients, and a pathological reaction pattern was induced in $44 \%$ of the patients by head-up tilt testing. In comparison, non-pathological findings were present in $56 \%$ of the patients. The $10 \%$ of patients who suffered from postural tachycardia syndrome will not be discussed further in this paper.

\section{Prodromal symptoms}

The medical histories of typical prodromal symptoms of syncopal events revealed the following results for the group without pathological findings during head-up tilt testing: $67 \%$ suffered from vertigo, $48 \%$ had nausea, $46 \%$ had black eyesight, $35 \%$ presented sweating, $28 \%$ experienced headache or head pressure, $14 \%$ exhibited tachycardia and $7 \%$ exhibited bradycardia. Thirty percent of these patients had at least one injury (a significant contusion, bone fracture, cut, commotio cerebri, or contusio cerebri) as the result of falling to the ground during a syncopal event. Head injuries represented approximately $47 \%$ of all injuries.

Patients with a syncopal event during head-up tilt testing reported vertigo in $82 \%$ of the cases as a typical prodromal symptom. Nausea was reported by $58 \%$, black eyesight by $55 \%$, sweating by $52 \%$, headache or head pressure by $29 \%$, tachycardia by $14 \%$ and bradycardia by $11 \%$. Approximately $47 \%$ of these patients presented injuries that were caused by accidents during syncope. Head injuries represented $55 \%$ of all injuries.

Significance was found for more frequent occurrence of the prodromal symptoms sweating and vertigo and of injuries in patients who experienced a syncopal event during head-up tilt testing (Figs. 1 and 2).

\section{Accompanying diseases}

The distribution pattern of accompanying diseases showed significantly more frequent occurrence of accompanying diseases in the group without pathologies during head-up tilt testing than in the group with induced syncope $(P<0.003)$. Whereas $63 \%$ of the asymptomatic patients had at least one accompanying disease, only $41 \%$ of the patients in the group with positive test results had at least one accompanying disease (Fig. 2).

\section{Total count of syncopal events}

The average patient not experiencing syncope during head-up tilt testing had previously suffered from $2.4 \pm 3$ (SD) syncopal events during his or her life. However, patients experiencing syncope during testing reported histories of $6.3 \pm 8$ (SD) syncopal events in their medical history. This highly significant difference $(P<0.001)$ remained stable even after 3 patients with extremely high numbers of previous syncopal events were removed

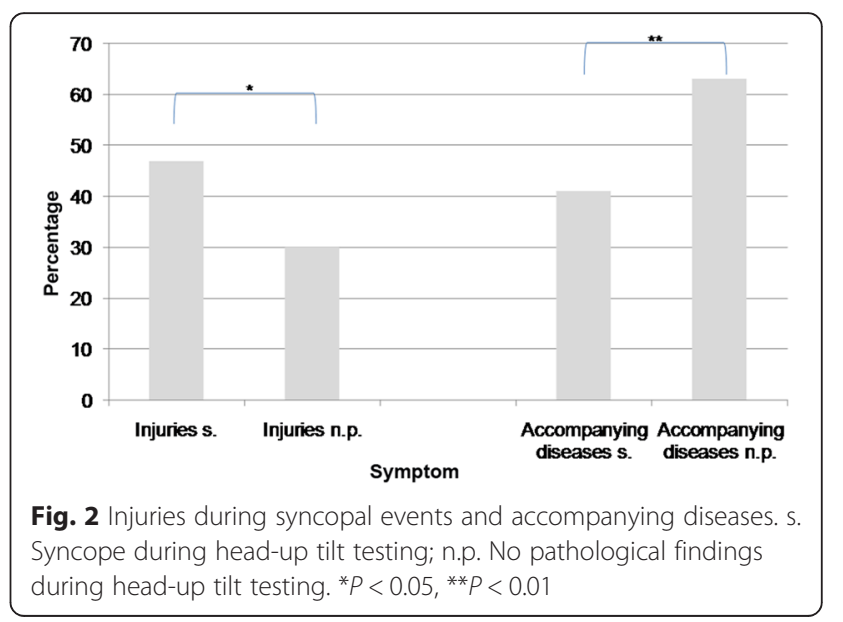


from the analysis. After this correction, the mean value for patients with syncope during head-up tilt testing was $4.059 \pm 3$ (SD) $(P<0.002)$ (Fig. 3). No significant difference in the total number of syncopal events was found between male and female patients.

\section{Height and body mass index}

The average height of female patients not experiencing syncope during head-up tilt testing was $162 \pm 6$ (SD) cm, whereas that of asymptomatic males was $175 \pm 6$ (SD) $\mathrm{cm}$. The mean value of height in symptomatic females was $167 \pm 5$ (SD) cm, whereas that of symptomatic males was $180 \pm 7$ (SD) $\mathrm{cm}$. Patients who developed syncope during testing were significantly taller $(P<0.002$ for females, $P<0.032$ for males).

In patients without syncope during head-up tilt testing, mean body mass index was $24 \pm 4$ (SD) $\mathrm{kg} / \mathrm{m}^{2}$ for females and $27 \pm 3$ (SD) $\mathrm{kg} / \mathrm{m}^{2}$ for males. In patients with syncope, mean body mass index was $24 \pm 3$ (SD) $\mathrm{kg} / \mathrm{m}^{2}$ for females and $26 \pm 3$ (SD) $\mathrm{kg} / \mathrm{m}^{2}$ for males. No significant results were found when comparing patients who were symptomatic or asymptomatic during tilt table testing.

\section{Electrocardiography}

Intermittent cardiac arrhythmia was documented for three patients in the group without syncope during testing. The other patients in this group showed a normal sinusoidal rhythm. Without exceptions, the patients in the syncope group showed a sinusoidal rhythm in the 12-channelelectrocardiogram. During the ECG analyses, special attention was given to PQ-time as a possible predictor of bradycardia-like disturbances of heart rhythm and increased vagal tone. However, no significant differences were found between asymptomatic and symptomatic patients. The mean value of PQ-time was $166 \pm 51$ (SD) ms in patients without detectable syncope and $167 \pm 33$ (SD) $\mathrm{ms}(P=0.880)$ in patients with syncope during headup tilt testing.

\section{Discussion}

During military deployment, syncope is the most frequent reason for the referral of US-American soldiers to cardiology after ischemic evaluation and arrhythmia/palpitations [13]. Injuries associated with syncopal events are infrequently registered in soldiers [5].

In aviators, syncope is particularly problematic because syncopal events during flight can impair safety, possibly causing loss of the airplane and the crew's life $[3,14]$. In a previous study, vasovagal syncope and heart rhythm-associated syncope were found to be correlated with the sudden incapacitation of aviators [3]. Multi-pilot requirements can ensure higher aviation safety [3]. In a systematic review, the incidence of sudden aviator incapacitation was found to lie in the range of 0.19 to 0.45 times $/ 10^{6}$ flight hours [15]. Another study reported 39 in-flight incapacitations for U.S. airlines between 1993 and 1998. These incapacitations included 9 cases of loss of consciousness, 4 of which were due to vasovagal syncope and one of which was due to neurogenic syncope [16]. Therefore, screening military aviators for syncope is important to prevent in-flight incapacitation.

This study was designed to identify factors that predict positive tilt table testing results in patients with syncope. The prognostic significance of the examined parameters regarding the sensitivity of tilt table testing as a diagnostic tool for patients with syncope was very heterogeneous. The applied modified Westminster protocol is considered especially useful for syncope diagnosis without the underestimation of cardioinhibitory responses [12].

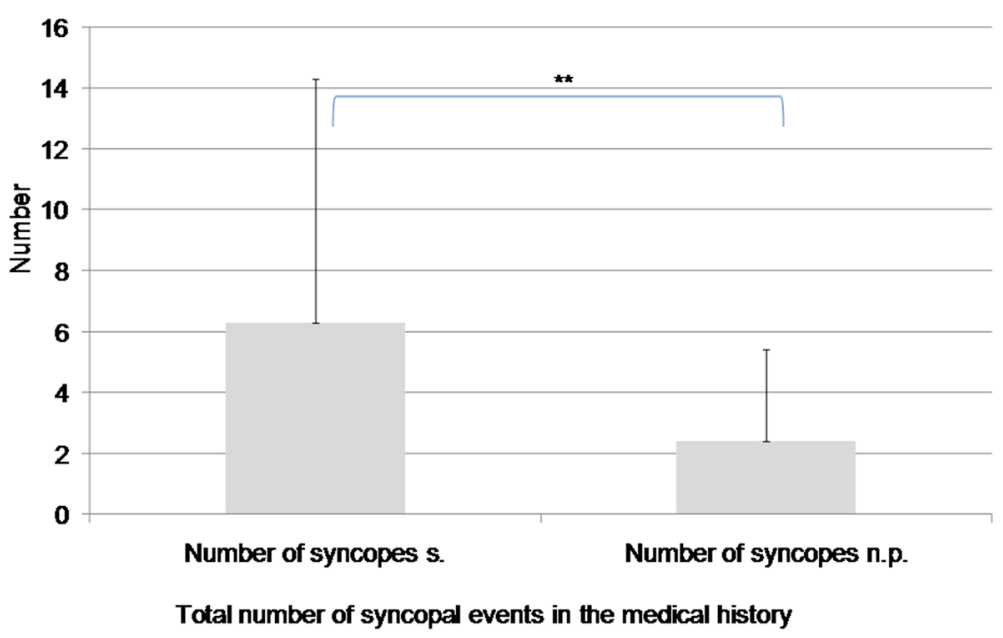

Fig. 3 Total count of syncopal events. s. Syncope during head-up tilt testing; n.p. No pathological findings during head-up tilt testing. ${ }^{* *} P<0.01$ 
The results presented here extend the current knowledge on the subject. Nausea, vertigo, sweating, headache or head pressure, epigastric symptoms and disturbed eyesight are typical prodromal symptoms of syncope [17]. History of syncope without heart disease accompanied by dizziness, nausea and diaphoresis is considered predictive of neurocardiogenic syncopal reactions [18]. Patients with a history of syncope often report visual blurring, dysesthesia, sighing dyspnea, tremor in fingers and diaphoresis. Sensations of hot flashes immediately before syncope are positively correlated with positive head-up tilt testing results. In contrast, the predictive value of clinical questionnaires appears negligible [19]. Hypertension is negatively correlated with head-up tilt testing results [12]. Low blood pressure and increased plasma renin levels predict orthostatic intolerance in dehydrated subjects [20]. The lack of an early sympathetic over-reactivity that leads to an increase in heart rate also predicts negative head-up tilt table test results [21]. The prediction of a forthcoming syncope during head-up tilt testing based on constant measurement of blood pressure and heart rate is possible with acceptable sensitivity and specificity [22]. The measurement of baroreceptor sensitivity and heart rate variability using power spectral analysis might contribute to an increased sensitivity of tilt testing in children [23]. The rate of positive results found during passive tilt testing can be tremendously increased by chemical agents, such as sublingual nitroglycerine. This agent appears to effectively unmask syncope with only a minor decrease in specificity [24]. However, reactions to nitroglycerin are partially age-dependent, and a more gradual decline of blood pressure is found in elderly patients [25]. Gender, presence of structural heart disease, baseline heart rhythm and presenting symptoms before the test were found to be of no important predictive value [26].

The medical history of prodromal symptoms yields very important hints concerning the causes of syncope. Nevertheless, approximately $30 \%$ of syncopal events occur without any prodromal symptoms [27]. If typical prodromal symptoms are absent, obtaining the correct diagnosis is much more difficult [28].

In the analysis presented here, significant differences were found for vertigo and sweating between patients who expressed syncope during head-up tilt testing and patients who did not.

Further, injuries due to syncopal events were significantly more frequent in patients with positive test results. This finding agrees well with previous reports [29]. The high percentage of serious injuries found here for both groups underlines the importance of accurate diagnoses and therapeutic strategies for syncope patients. Other authors reported frequencies of injuries per syncopal event of up to $35 \%$ in the elderly. Serious injuries are defined as bone fractures, subdural hematomas and traffic accidents caused by syncope. The proportion of such serious events is $17 \%$ [30, 31].

Importantly, no significant differences were identified for nausea, headache or head pressure, and black eyesight. Tachycardia and bradycardia were rarely experienced by patients with negative or positive head-up tilt testing results as prodromal symptoms. No significant difference was found between the two groups in this respect. However, this does not necessarily mean that these symptoms are not present. Due to adaptation, the patients might simply be unaware of these symptoms. This lack of awareness renders tachycardia and bradycardia of little use as diagnostic criteria based on medical history.

Comparison of the accompanying diseases also revealed significant differences between the patients yielding positive and negative test results. An increasing number of accompanying diseases appears to be negatively correlated with the number of negative head-up tilt testing results. Increasing age is usually associated with an increasing number of chronic diseases. Indeed, previous analyses have shown that the risk of syncopal events that are not reproducible by head-up tilt testing is especially increased in elderly patients. Possible reasons include age-related physiological changes and an increasing number of chronic diseases and the associated pharmaceutical therapy [32]. In contrast, young age is considered an independent predictor of positive results during head-up tilt testing [29]. Other authors deny the predictive value of age. However, it is widely accepted that syncope typically occurs in young patients during earlier stages of testing than in older patients [26]. Nevertheless, head-up tilt testing shows satisfying results for the differential diagnosis of etiologically unclear syncope in patients aged over 65 years, and the results are even more satisfying in patients aged over 80 years [32].

Next to its effects on the reliability of tilt testing, age also influences the occurrence of syncope. As demonstrated in a recent review [33], the average incidence of syncope increases markedly in patients older than 70 years. Of note, studies addressing orthostatic intolerance in non-clinical subjects, both generally and for soldiers in particular $[2,5,13,14]$, usually analyze much younger cohorts than that assessed here. Accordingly, this analysis might overestimate the overall sensitivity of head-up tilt testing, and this sensitivity might be considerably lower in younger patients.

The finding that patients with a high number of syncopal events in their medical history are more likely to show syncope during tilt testing is consistent with previous literature [9]. A history of neurocardiogenic syncope is positively correlated with the results of tilt testing [12, 26]. In addition, the risk of remitting syncope is significantly increased if the number of previous syncopal events is 
raised from one to three. This risk is further influenced by the frequency of the events. Patients who have experienced repeated syncope over a short period present a higher risk than patients with longer periods between syncopal events [34-36]. The probability of experiencing further syncopal events after a single event is approximately $30 \%$ [37].

The identification of physiognomic differences between patients with positive and negative head-up tilt testing results was a further aim of the study. The two groups were different in height. Patients with syncope induced by head-up tilt testing were significantly taller than patients with negative test results. This was true for both male and female patients. In contrast, no significant difference was found in body mass index for both men and women. Although no significant difference was found between the groups, patients with syncope were likely to have body mass indices at borderline obesity levels in this study, irrespective of the head-up tilt testing results. An asthenic-leptosomal physique (which is common in patients with arterial hypotonia) was not indicated as a possible predisposition based on the data obtained here. In contrast, other authors have reported a negative correlation between body mass index and head-up tilt testing responses [12].

PQ-time as a possible indicator of bradyarrhythmic heart rhythm disturbances and increased vagal tone was of special interest while interpreting the 12-channelelectrocardiogram charts. In particular, the cardioinhibitory variant of neurocardiogenic syncope has been reported to be associated with sinusoidal bradycardia up-to asystolia of more than 30 seconds or $\mathrm{II}^{\circ}$ atrioventricular blocks [38]. Further, the presence of a junction rhythm is an independent predictor of positive head-up tilt testing results [29]. However, we did not observe any prolongation of PQ-time, and the symptomatic and asymptomatic patients during head-up tilt testing did not show any significant difference. Admittedly, the meaningfulness of the results is limited. Permanent electrocardiography measurement would have been a more meaningful method, an undeniable limitation of the study.

Of note, short time between the last syncope and headup tilt testing is considered a further predictor of positive test results [29]. However, this element of the patients' medical histories was not analyzed in this study.

Restriction of food and water intake might have affected the probability of positive head-up tilt testing results due to dehydration. These restrictions were applied to standardize the test conditions.

Head-up tilt testing was performed in the morning to ensure the standardization of this proof-of-principle analysis. The low number of participants did not allow the patients to be divided into groups for analyses at different times of day. Several studies suggest that the vasovagal response to head-up tilt exhibits a circadian rhythm, showing a higher frequency of syncopal episodes in the morning [39-43]. Therefore, head-up tilt testing was performed during the morning to increase the likelihood of obtaining positive test results.

In future studies more differentiated analyses should be performed, generally dividing the volunteers into male and female groups. The low number of volunteers used in this proof-of-principle study does not allow meaningful assessments regarding this issue. Therefore, gender stratification was only performed for body mass index (BMI) and body height, which are considerably different in men and women and could be calculated for all volunteers. As previously observed by us, the total incidence of syncope is similar between genders [33].

Despite the existence of considerable knowledge regarding the occurrence of syncope in soldiers [2, 5, 13], associations between various aspects of military duty or deployment and syncope are poorly understood, with the exception of standing in ranks [2]. Future studies should also focus on this aspect.

\section{Conclusion}

In summary, the following criteria were suggested for the ideal head-up tilt test patient:

Main criteria:

- Three or more syncopal events in the patient's medical history

- Typical prodromal symptoms (especially vertigo, sweating or injuries in association with syncopal events).

Minor criteria:

- No further accompanying diseases

- Body height in the upper range and a body mass index in the upper normal range

Immediate head-up tilt testing might be advisable if at least one main and one minor criterion are satisfied. Admittedly, the low sample size and wide age ranges used in this proof-of-principle assessment do not provide sufficient statistical power for creating mathematical models of syncope risk. Neither the applied statistical tests nor the sample size allow for the reliable mathematical determination of criteria to identify patients who are at greater risk of syncope. Future confirmatory studies with sufficient power are warranted to confirm the suggested criteria, to ensure that the calculations are robust, and to allow for gender stratification. Such future studies should also control for underlying medical conditions, which might be critical for the outcome. 
Nevertheless, head-up tilt testing should be the first diagnostic procedure used for patients with typical medical histories rather than the last test procedure undertaken. Appropriate diagnostic infrastructure and qualifications are advisable for diagnosing armed forces medical service personnel, especially in view of the importance of syncope within military settings and with respect to aviation safety $[2,5,13,14]$.

\section{Availability of supporting data}

All relevant data are presented in the paper.

\section{Abbreviations}

BMI: body mass index; ECG: electrocardiogram; ECG: electrocardiographic; POTS: Postural tachycardia syndrome; SD: Standard deviation.

\section{Competing interests}

The authors declare that there is no competing interest according to the guidelines of the International Committee of Medical Journal Editors.

\section{Authors' contributions}

HJG was responsible for the study design and participated in writing the manuscript. LMH participated in writing the manuscript. FG participated in writing the manuscript. HF participated in writing the manuscript. SAJ analyzed the data and participated in writing the manuscript. All authors have read and approved the final manuscript.

\section{Author details}

${ }^{1}$ The Practice of Dr. Jungblut, Frankfurt/Main, Germany. ${ }^{2}$ The Flight Medicine Clinic at Fassberg, German Armed Forces, Faßberg, Germany. ${ }^{3}$ The Department of Tropical Medicine at the Bernhard Nocht Institute, German Armed Forces Hospital of Hamburg, Hamburg, Germany. ${ }^{4}$ The Department of Microbiology, Virology and Hygiene, University Medicine Rostock, Rostock, Germany.

Received: 11 February 2015 Accepted: 27 November 2015

Published online: 02 December 2015

\section{References}

1. Thijs RD, Wieling W, Kaufmann H, van Dijk JG. Defining and classifying syncope. Clin Auton Res. 2004;14:4-8.

2. Fitch DR, Rippert ET. Syncope in military formations: a persistent problem. Mil Med. 1992:157:577-8.

3. Evans S, Radcliffe SA. The annual incapacitation rate of commercial pilots. Aviat Space Environ Med. 2012:83:42-9.

4. Verordnung (EU) Nr. 1178/2011 der Kommission vom 3. November 2011 zur Festlegung technischer Vorschriften und von Verwaltungsverfahren in Bezug auf das fliegende Personal in der Zivilluftfahrt gemäß der Verordnung (EG) Nr. 216/2008 des Europäischen Parlaments und des Rates, Anhang IV Teil MED Abschnitt A 030 Tauglichkeitszeugnisse sowie Abschnitte B und C - Retrieved from: http://eur-lex.europa.eu/LexUriServ/ LexUriServ.do?uri=OJ:L:2011:311:0001:0193:DE:PDF. Accessed 2 Sep 2015. [Text in German].

5. Armed Forces Health Surveillance Center (AFHSC). Syncope, active and reserve components, U.S. Armed Forces, 1998-2012. MSMR. 2013;20:5-9.

6. Deharo JC, Peyre JP, Ritter PH, Chalvidan T, Le Tallec L, Djiane P. Treatment of malignant primary vasodepressive neurocardiogenic syncope with a rate responsive pacemaker driven by heart contractility. PACE. 1998;21:2688-90.

7. Kenny RA, Bayliss J, Ingram A, Sutton R. Head-up tilt: A useful test for investigating unexplained syncope. Lancet. 1986;1:1352-4.

8. Fitzpatrick AP, Sutton R. Tilting towards a diagnosis in recurrent unexplained syncope. Lancet. 1989;1:658-60.

9. Raviele A, Gasparini G, Di Pede F, Delise P, Bonso A, Piccolo E. Usefulness of head-up tilt in evaluation of patients with syncope of unknown origin and negative electrophysiologic study. Am J Cardiol. 1990:65:1322-7.

10. Jungblut S, Frickmann H, Römer A, Gilfrich HJ. Importance of tilt-table testing compared with Schellong's test for the diagnosis of orthostatic dysregulations/syncopes. Med Klin (Munich). 2006;101:198-202.
11. Zanchetti A, Mancia G. The centenary of blood pressure measurement: A tribute to Scipione Riva-Rocci. J Hypertension. 1996;14:1-12.

12. Lelonek M, Stanczyk A, Goch JH. Effect of passive tilting duration on the outcome of head-up tilt testing. Acta Cardiol. 2007;62:547-52.

13. Sullenberger L, Gentlesk PJ. Cardiovascular disease in a forward military hospital during operation iraqi freedom: a report from deployed cardiologists. Mil Med. 2008;173:193-7.

14. Carter D, Pokroy R, Grossman A, Azaria B, Goldstein L. Head-up tilt test for recurrent syncope in pilots. Aviat Space Environ Med. 2005;76:1167-9.

15. Huster KM, Muller A, Prohn MJ, Nowak D, Herbig B. Medical risks in older pilots: a systematic review on incapacitation and age. Int Arch Occup Environ Health. 2014;87:567-78.

16. DeJohn CA, Wolbrink AM, Larcher JG. In-flight Medical Incapacitation and Impairment of U.S. Airline Pilots: 1993 to 1998. Flight Safety Digest. 2005;24:1-23.

17. Saul JP. Syncope: Etiology, Management and when to refer. J S C Med Assoc. 1999:95:385-7

18. Asensio E, Oseguera J, Loría A, Gómez M, Narváez R, Dorantes J, et al. Clinical findings as predictors of positivity of head-up tilt table test in neurocardiogenic syncope. Arch Med Res. 2003;34:287-91.

19. Vallejo M, Hermosillo AG, Márquez MF, Urquidez AK, Sotomayor A

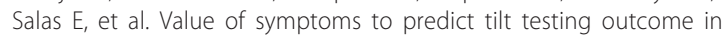
patients with clinical suspicion of vasovagal syncope. Arch Med Res. 2007;38:579-83.

20. Harrison MH, Kravik SE, Geelen G, Keil L, Greenleaf JE. Blood pressure and plasma renin activity as predictors of orthostatic intolerance. Aviat Space Environ Med. 1985:56:1059-64.

21. Movahed MR. Predictor of a negative tilt-table test result based on early heart rate variation in patients with a history of syncope. Am Heart Hosp J. 2007;5:247-9.

22. Virag N, Sutton R, Vetter R, Markowitz T, Erickson M. Prediction of vasovagal syncope from heart rate and blood pressure trend and variability: experience in 1,155 patients. Heart Rhythm. 2007:4:1375-82.

23. Dalla Pozza R, Kleinmann A, Kozlik-Feldmann R, Netz H. Tilt table testing in childhood: improved sensitivity by non-invasive haemodynamic monitoring devices? Acta Cardiol. 2006:61:615-21.

24. Foglia-Manzillo G, Giada F, Fteita N, Nessi I, Santarone M, Raviele A. Tilt testing potentiated with sublingual nitroglycerin in children with unexplained syncope. Eur Heart J. 2007;28:2605-9.

25. Verheyden B, Gisolf J, Beckers F, Karemaker JM, Wesseling KH, Aubert AE, et al. Impact of age on the vasovagal response provoked by sublingual nitroglycerine in routine tilt testing. Clin Sci (Lond). 2007;113:329-37.

26. Kazemi B, Haghjoo M, Arya A, Sadr-Ameli MA. Predictors of response to the head-up tilt test in patients with unexplained syncope or presyncope. Pacing Clin Electrophysiol. 2006;29:846-51.

27. Morillo CA, Villar JC. Neurocardiology. Neurogenic syncope. Baillieres Clin Neurol. 1997;6:357-80.

28. Sutton R, Bloomfield DM. Indications, methology and classification of results of tilt-table testing. Am J Cardiol. 1999:84:10-9.

29. Oh JH, Kim JS, Kwon HC, Hong KP, Park JE, Seo JD, et al. Predictors of positive head-up tilt test in patients with suspected neurocardiogenic syncope or presyncope. Pacing Clin Electrophysiol. 2003;26:593-8.

30. Day SC, Cook EF, Funkenstein H, Goldman L. Evaluation and outcome of emergency room patients with transient loss of consciousness. American J Med. 1982;73:15-23.

31. Kapoor WN. Evaluation and outcome of patients with syncope. Medicine. 1990;69:160-75.

32. Bloomfield D, Maurer M, Bigger JT. Effects of age on outcome of tilt table testing. Am J Cardiol. 1999;83:1055-8.

33. Da Silva RM. Syncope: epidemiology, etiology, and prognosis. Front Physiol. 2014:5:471.

34. Natale A, Geigler MJ, Maglio C, Newby KH, Dhala A, Akhtar M, et al. Recurrence of neurocardiogenic syncope without pharmacologic interventions. Am J Cardiol. 1996;77:1001-3.

35. Sheldon R, Rose S, Flanagan P, Koshman ML, Killam S. Risk factors for syncope recurrence after a positive tilt-table test in patients with syncope. Circulation. 1996;93:973-81.

36. Grimm W, Degenhardt M, Hoffmann J, Menz V, Wirths A, Maisch B. Syncope recurrence can better be predicted by history than by head-up tilt testing in untreated patients with suspected neurally mediated syncope. Eur Heart J. 1997;18:1465-9. 
37. Kapoor WN, Peterson J, Wieand HS, Karpf M. Diagnostic and prognostic implications of recurrences in patients with syncope. Am J Med. 1987;83:700-8

38. Folino AF, Buja GF, Martini B, Miorelli M, Nava A. Prolonged cardiac arrest and complete AV block during up-right tilt test in young patients with syncope of unknown origin - prognostic and therapeutic implications. Eur Heart J. 1992;13:1416-21.

39. Hu K, Scheer FA, Laker M, Smales C, Shea SA. Endogenous circadian rhythm in vasovagal response to head-up tilt. Circulation. 2011;123:961-70.

40. Lewis NC, Atkinson G, Lucas SJ, Grant EJ, Jones H, Tzeng YC, et al. Diurnal variation in time to presyncope and associated circulatory changes during a controlled orthostatic challenge. Am J Physiol Regul Integr Comp Physiol. 2010;299:R55-61.

41. Zoghi M, Duygu H, Gungor H, Nalbantgil S, Ozerkan F, Akilli A, et al. Circadian and infradian rhythms of vasovagal syncope in young and middle-aged subjects. Pacing Clin Electrophysiol. 2008;31:1581-4.

42. Van Dijk N, Boer MC, De Santo T, Grovale N, Aerts AJ, Boersma L, et al. Daily, weekly, monthly, and seasonal patterns in the occurrence of vasovagal syncope in an older population. Europace. 2007:9:823-8.

43. Kula S, Olguntürk R, Tunaoğlu FS, Saygili A. Distribution of syncopal episodes in children and adolescents with neurally mediated cardiac syncope through the day. Europace. 2005;7:634-7.

\section{Submit your next manuscript to BioMed Central} and we will help you at every step:

- We accept pre-submission inquiries

- Our selector tool helps you to find the most relevant journal

- We provide round the clock customer support

- Convenient online submission

- Thorough peer review

- Inclusion in PubMed and all major indexing services

- Maximum visibility for your research 